\title{
CLOSED SUBSPACES OF FINITE CODIMENSION IN SOME FUNCTION ALGEBRAS
}

\author{
RAMESH V. GARIMELLA AND N. V. RAO
}

(Communicated by John B. Conway)

\begin{abstract}
We characterize all closed subspaces of finite codimension in some specific types of function algebras e.g. these include $C(X)$ : algebra of continous functions on a compact Hausdorff space, $C^{n}[a, b]$ : the algebra of $n$-times continuously differentiable functions on the closed interval $[a, b]$. Our work is a generalization of the well-known Gleason-Kahane-Želazko theorem $[\mathbf{3}, \mathbf{6}]$ for subspaces of codimension one in arbitrary unitary Banach algebras.
\end{abstract}

0. Introduction. In [5], K. Jarosz settled a conjecture of Warner and Whitley [10] by proving the following: Let $S$ be a compact Hausdorff space such that each point of $S$ is a $G_{\delta}$-set and $M$ be a closed subspace of finite codimension in $C(S)$, the algebra of continuous functions on $S$ with the sup norm. Let $k$ be any positive integer. Suppose that for every $f$ in $M, f$ has a least $k$ distinct zeroes in $S$. Then there exist $k$ distinct points $s_{1}, s_{2}, \ldots, s_{k}$ in $S$ such that for every $f$ in $M$; $f\left(s_{1}\right)=f\left(s_{2}\right)=\cdots=f\left(s_{k}\right)=0$.

In this paper, we generalize this theorem as follows.

THEOREM 3.1. Let $X$ be a compact Hausdorff space and $M$ be a closed subspace of finite codimension in $C(X)$ such that for every $f$ in $M, f(x)=0$ for some $x$ in $X$. Then (a) There exists an $x_{0}$ in $X$ such that $f\left(x_{0}\right)=0$ for every $f$ in $M$; (b) there exists finitely many points $x_{1}, x_{2}, \ldots, x_{k}$ such that $f\left(x_{j}\right)=0$ for $1 \leq j \leq k$ and these are the only common zeroes of $M$ in the following sense: If $C$ is any compact $G_{\delta}$-set containing $x_{1}, x_{2}, \ldots, x_{k}$, then there exists an $f$ in $M$ such that $f(x) \neq 0$ for every $x$ in $X \backslash C$.

Also, in this paper, we prove the polynomial lemma.

LEMMA 1.1. Let $K$ be any closed subset of the complex plane such that $K^{0}=\varnothing$. Let $p_{j}, 1 \leq j \leq k$, be nonconstant polynomials of one complex variable $z$. Further assume $\sum_{j=1}^{k} w_{j} p_{j}$ has a zero in $K$ for any given complex numbers $w_{j}, 1 \leq j \leq k$. Then there exists a $z_{0}$ in $K$ such that $p_{j}\left(z_{0}\right)=0$ for $1 \leq j \leq k$.

This is a generalization of a lemma in [5] and this allows us to obtain theorems of type 3.1 for algebras $C^{n}[a, b], \mathcal{L}^{1}(R)$ as well as $C(X)$. Recently C. P. Chen and P. J. Cohen [2] have proved that conclusions of Theorem 3.1 hold for selfadjoint regular $n$-point spectral Banach algebras. These include $C(X)[5]$ and also $L^{1}(G)$ where $G$ is any locally compact metrizable abelian group. Thus this solves both

Received by the editors March 24, 1986 and, in revised form, August 15, 1986.

1980 Mathematics Subject Classification (1985 Revision). Primary 40J15; Secondary 40J10, 40 J20.

Key words and phrases. $G_{\delta}$-set, polynomial ring, irreducible polynomial, Unique factorization domain, function algebras, ideals of finite codimension. 
conjectures of Warner and Whitley [10]. But [2 and 5] do not cover algebras of type $C^{n}[a, b]$, because these are not spectral. One of us in [8] has removed the condition of spectrality in the Chen and Cohen theorem, which then applies to algebras of $n$-times continuously differentiable functions. The results in this paper have some overlap with results from [2, $\mathbf{5}$ and $\mathbf{8}$ ], but the method given here is different and more direct. Further, our results apply to a more general class of algebras e.g., Fréchet algebras. For related results, refer to $[\mathbf{1}, \mathbf{3}, \mathbf{6}$ and $\mathbf{9}]$.

1. Proof of the polynomial Lemma 1.1 (as stated in the introduction). Suppose that $p_{j}(z), 1 \leq j \leq k$, have no common zeroes in $K$. If $p_{0}(z)$ is the greatest common divisor of $p_{j}, 1 \leq j \leq k$; by our supposition, $p_{0}$ does not vanish on $K$. Thus replacing $p_{j}$ by $p_{j} / p_{0}$, we can assume that any complex linear combination of $p_{j}$ has a zero in $K$ but $\left\{p_{j}\right\}$ do not have a common zero in the complex plane.

Let $\mathcal{P}(z, w)=\sum_{j=1}^{k} w_{j} p_{j}(z)$ where $w$ denotes the $k$ tuple $\left(w_{1}, w_{2}, \ldots, w_{k}\right) ; R$ denotes the polynomial ring over $\mathbf{C}$ in $k$ indeterminates $w_{1}, w_{2}, \ldots, w_{k} ; Q$ be the quotient field of $R$; and $R[z], Q[z]$ be the polynomial rings in one indeterminate $z$ over the rings $R$ and $Q$ respectively.

We claim that $P(z, w)$ is irreducible in the ring $R[z]$. If not, there exist polynomials $P_{1}(z, w), P_{2}(z, w)$ nonconstant and belonging to $R[z]$ so that $P(z, w)=$ $P_{1}(z, w) P_{2}(z, w)$. We can write

$$
P_{i}(z, w)=\sum_{0 \leq|j| \leq N} p_{i j} w^{j}
$$

where $w^{j}=w_{1}^{j_{1}} w_{2}^{j_{2}} \cdots w_{k}^{j_{k}}, j$ is the multi-index $\left(j_{1}, j_{2}, \ldots, j_{k}\right),|j|=\sum_{\lambda=1}^{k} j_{\lambda}$, $p_{i, j}(z)$ is a polynomial in $z$ with constant coefficients for $i=1,2$. Thus we consider $P_{i}(z, w)$ as polynomials in $w$ over the integral domain $\mathbf{C}[z]$. Since $\mathcal{P}(z, w)$ is a linear polynomial in $w_{j}$; we see that at least one of $P_{1}, P_{2}$ must be independent of $w$. Let us assume $P_{1}$ to be independent of $w$ and $P_{2}$ to be linear in $w$.

Let $P_{1}(z)=P_{1}(z, w)$. Thus $p_{j}(z)=P_{1}(z) \cdot p_{2, j}(z), 1 \leq j \leq k$, that is, $p_{j}(z)$ have a common divisor $P_{1}(z)$. By our assumption, they do not have any common divisor except constants and on the other hand $P_{1}(z)=P_{1}(z, w)$ is nonconstant, which is a contradicition. Hence $P(z, w)$ is irreducible. Also, $P(z, w)$ is primitive as a polynomial over $R$ by the same reasoning.

We now show that $P(z, w)$ is irreducible in the ring $Q[z]$. If not, then $P(z, w)=$ $P_{1}(z, w) P_{2}(z, w)$ where $P_{1}, P_{2}$ are nonconstant polynomials in $z$ with coefficients in $Q$. Since $R$ is a unique factorization domain $\left[11\right.$, p. 45] we can find $\alpha_{1}, \alpha_{2}, \beta_{1}, \beta_{2}$ in $R$ such that $\alpha_{1}, \alpha_{2}$ are coprime; $\beta_{1}, \beta_{2}$ are coprime and

$$
\frac{\alpha_{1}}{\alpha_{2}} \rho_{1}, \quad \frac{\beta_{1}}{\beta_{2}} \text { P belong to } R[z]
$$

and are primitive polynomials. By Gauss' Lemma [11, p. 46], the product of primitive polynomials is primitive and so

$$
\frac{\alpha_{1} \beta_{1}}{\alpha_{2} \beta_{2}} P(z, w)
$$

is a primitive polynomial. Since $P$ is also primitive, we see that $\alpha_{1} \beta_{1} / \alpha_{2} \beta_{2}$ is a unit and since $\alpha_{1} \beta_{1}, \alpha_{2} \beta_{2}$ are coprime, all of $\alpha_{1}, \beta_{1}, \alpha_{2}, \beta_{2}$ are units in the ring $R$ 
i.e. they are constants. This means that $P_{1}, P_{2}$ belong to $R[z]$. But $P=P_{1} P_{2}$ is irreducible in $R[z]$, which is a contradiction. Hence $P$ is irreducible in $Q[z]$. But then since $Q[z]$ is a Euclidean domain there exist $A, B$ in $Q[z]$ such that

$$
A P+B \frac{\partial \mathcal{P}}{\partial z}=1
$$

Thus there exists a nonzero $D$ in $R$ such that $D A, D B$ belong to $R[z]$ and

$$
D A P+D B \frac{\partial P}{\partial z}=D
$$

i.e.

$$
A_{1} P+B_{1} \frac{\partial P}{\partial z}=D
$$

Since $D$ is a polynomial in $w_{j}$, we can choose a ball $\Omega$ in $\mathbf{C}^{k}$ on which $D(w) \neq$ 0 . Thus for every $w$ in $\Omega$, the polynomial $\mathcal{P}(z, w)$ has $k$ distinct roots $z_{j}(w)$, $1 \leq j \leq k$, and we may assume that $z_{j}$ are holomorphic in $\Omega$ [4, p. 11]. Let $S_{j}=\left\{\omega \in \Omega ; z_{j}(\omega) \in K\right\}$. One of the $z_{j}$ belongs to $K$ for any given $w$ in $\Omega$. So $\Omega=\bigcup S_{j}$. Since each $S_{j}$ is closed in $\Omega$, one of the $S_{j}$ say $S_{1}$, must have nonempty interior. By the open mapping theorem, we see that $z_{1}(w)$ is locally constant on $S_{1}$ or $z_{1}\left(S_{1}\right)$ has nonempty interior. But $K^{0}=\varnothing$. Thus $z_{1}$ is constant on $\Omega$. Hence there exists a $z_{0}$ such that $P\left(z_{0}, w\right) \equiv 0$ on $\Omega$ and by analytic continuation on all of $\mathbf{C}^{k}$. Thus $p_{j}\left(z_{0}\right)=0$ for $1 \leq j \leq k$, which is a contradiction. This proves Lemma 1.1 .

COROLlARY 1.2. If $M$ is any subspace of the polynomial algebra $\mathbf{C}[z]$ and $Z$ is the set of common zeroes of $M$ allowing multiplicity, then there exists a polynomial $p$ in $M$ such that $p \neq 0$ on $K \backslash Z$.

Proof. Suppose that for every $p$ in $M, p$ has a zero in $K$. Let $Z_{p}$ be the set of zeroes of $p$ in $K$ counting multiplicity. Then by Lemma 1.1, the family of $\left\{Z_{p} ; p \in M\right\}$ is family of compact sets with the finite intersection property. Hence $Z=\bigcap_{p \in M} Z_{p} \neq \varnothing$. Let $Z=\left\{z_{j}, 1 \leq j \leq k\right\}$ and $q=\left(z-z_{1}\right)\left(z-z_{2}\right) \cdots\left(z-z_{k}\right)$; $M_{0}=\{p \in \mathbf{C}[z] ; q p \in M\}$. Now if every $p$ in $M_{0}$ has a zero in $K$, once again by Lemma 1.1 , there exists a $z_{0}$ in $K$ such that $p\left(z_{0}\right)=0$ for every $p$ in $M_{0}$. But then $z_{0}$ is a common zero of $M$ and so $z_{0}$ belongs to $Z$. This increases the multiplicity of $z_{0}$ for $M$, which is a contradiction. Thus there exists a $p_{0}$ in $M_{0}$ such that $p_{0} \neq 0$ on $K$ and $q p_{0}$ belongs to $M$ and $q p_{0} \neq 0$ in $K \backslash Z$.

\section{Applications.}

THEOREM 2.1. Let $K$ be a closed subset of $C$ such that $K^{0}=\varnothing$. Let $A$ be an algebra of functions on $K$ provided with a locally convex Hausdorff topological vector space structure in which evaluation at any point in $K$ is continuous on $A$. Suppose that $M$ is a subspace of $A$ and $P$ is the algebra of polynomials in $z$. If for every $f$ in $M$, there exists a $z$ in $K$ such that $f(z)=0$ and $P \cap M$ is dense in $M$; then the set $Z$ of common zeroes of $M$ is finite and nonempty, and there exists an $f$ in $M$ such that $f \neq 0$ on $K \backslash Z$.

PROOF. By our assumptions and the polynomial lemma, we obtain that functions in $P \cap M$ have at least one common zero. Since point evaluations are continuous and $P \cap M$ is dense in $M$, all functions in $M$ have at least one common zero. 
So the set $Z$ of common zeroes of $M$ is finite and nonempty. Further, by Corollary 1.2 , there exists a $p$ in $P \cap M$ such that $p \neq 0$ on $K \backslash Z$. Q.E.D.

COROLlaRY 2.2. Let $K=[a, b]$, a closed interval in the real line, and $C^{n}(K)$ be the algebra of $n$-times continuously differentiable functions with the standard Banach algebra structure. Suppose $M$ is a closed subspace of $C^{n}(K)$ of finite codimension. Then the set $Z$ of all common zeroes of $M$ is finite (possibily empty) and there exists a fucntion $f$ in $M$ such that $f \neq 0$ in $K \backslash Z$.

PROOF. Let $P$ be the algebra of polynomials in $z$. Since $M$ is of finite codimension and $P$ is dense in $C^{n}(K), P \cap M$ is dense in $M$. Applying Theorem 2.1, we obtain the result.

REMARK 2.3. The proof above also applies to $C^{\infty}(R)$, the Fréchet algebra of $C^{\infty}$ functions on $R$.

COROLLARY 2.4. Let $A$ be the algebra of functions on $R$ which are the Fourier transforms of $L^{1}$-functions on $R$. If $f=\hat{g}$ is in $A$, we define $\|f\|=\|g\|_{1} ; A$ is a Banach algebra. Let $M$ be a closed subspace of finite codimension in $A$. If $Z$ is the set of common zeroes of $M$, then $Z$ is finite (possibly empty) and there exists an $f$ in $M$ such that $f \neq 0$ in $R \backslash Z$.

Proof. Let $\mathcal{P}=\left\{p\right.$ a polynomial; $\left.p(t) e^{-t^{2} / 2} \in M\right\}$ and $D=e^{-t^{2} / 2} P$. It is well known that $D$ is a dense subspace of $A$ and hence $D \cap M$ is dense in $M$. Thus $t$ is a common zero of $M$ if and only if it is a common zero of $D \cap M$ and hence of $P$. But by the polynomial lemma, it follows that if $Z$ is the set of common zeroes of $P$ allowing multiplicity, then $Z$ is finite (possibly empty) and there exists $p$ in $P$ such that $p(t) \neq 0$ on $R \backslash Z$. Q.E.D.

3. Proof of Theorem 3.1 (see the introduction for the formulation). By Theorem 1 of Jarosz [5], all functions in $M$ have a common zero in $X$. Since $M$ is of finite codimension and closed, there can only be finitely many common zeroes (say) $x_{1}, x_{2}, \ldots, x_{k}$. Also there exist finitely many Borel measures $\mu_{j}, 1 \leq j \leq l$, of bounded variation on $X$ such that $f$ belongs to $M$ if and only if $f\left(x_{j}\right)=0$ for $1 \leq j \leq k$ and $\int f d \mu_{j}=0$ for $1 \leq j \leq l$.

We may assume that $\left|\mu_{j}\right|(Z)=0$ for $1 \leq j \leq l$ where $Z$ is the set of all zeroes of $M$. Let

$$
\tilde{M}=\left\{f \in C(X) ; \int f d \mu_{j}=0 \text { for } 1 \leq j \leq l\right\} .
$$

By Theorem 1 of [5], either there exists an $f$ in $\tilde{M}$ such that $f \neq 0$ on $X$ or all functions in $\tilde{M}$ have a common zero $x_{0}$. But such an $x_{0}$ would belong to $Z$ and the Dirac measure $\delta_{x_{0}}$ would belong to the linear span of $\mu_{j}, 1 \leq j \leq l$. This is impossible since $\delta_{x}, x$ in $Z$, and $\mu_{j}, 1 \leq j \leq l$, are linearly independent. Hence there exists an $f$ in $M$ such that $f \neq 0$ on $X$.

Now let us choose $f_{j}$ so that $f_{j}(Z)=0$ and $\int f_{i} d \mu_{j}=\delta_{i j}, 1 \leq i, j \leq l$. Choose an $\varepsilon>0$ such that $|f|-\varepsilon \sum\left|f_{j}\right|>0$ on $X$ and an open neighborhood $V$ of $Z$ such that

$$
\left|\mu_{j}\right|(V)<\varepsilon / \operatorname{Max}_{x}\left(|f|-\varepsilon \sum\left|f_{j}\right|\right) .
$$

Choose a compact $G_{\delta}$ set $C_{0}$ such that $Z \subset C_{0} \subset V$ and a function $g$ in $C(X)$ such that $0 \leq g \leq 1, g=1$ only on $C_{0}, g=0$ outside $V$. This is possible only because $C_{0}$ is a $G_{\delta}$ set. 
Let $\psi=g\left(|f|-\varepsilon \sum\left|f_{j}\right|\right)$. Then

$$
\begin{aligned}
\left|\mu_{j} \psi\right| & \leq\left|\mu_{j}\right|(V) \operatorname{Max}|\psi| \\
& <\left(\varepsilon / \operatorname{Max}\left(|f|-\varepsilon \sum\left|f_{j}\right|\right)\right) \cdot \operatorname{Max}\left(|f|-\varepsilon \sum\left|f_{j}\right|\right) \\
& \leq \varepsilon .
\end{aligned}
$$

Consequently $f-\psi+\sum \mu_{j}(\psi) f_{j}=h$ satisfies

$$
|h| \geq|f|-|\psi|-\varepsilon \sum\left|f_{j}\right|=(1-g)\left(|f|-\varepsilon \sum\left|f_{j}\right|\right)
$$

and so $|h|>0$ on $X \backslash C_{0}$. Further $h(Z)=0, \int h d \mu_{j}=-\mu_{j}(\psi)+\mu_{j}(\psi)=0$ for $1 \leq j \leq l$. So $h$ belongs to $M$. Q.E.D.

\section{REFERENCES}

1. C. P. Chen, A generalization of the Gleason-Kahane-Želazko theorem, Pacific J. Math. 107 (1983), 81-87.

2. C. P. Chen and P. J. Cohen, Ideals of finite codimension in commutative Banach algebras (preprint).

3. A. M. Gleason, A characterization of maximal ideals, J. Analyse Math. 19 (1967), 171-172.

4. M. Herve, Several complex variables, local theory, Oxford Univ. Press and TIFR, 1963.

5. K. Jarosz, Finite codimensional ideals in function algebras, Trans. Amer. Math. Soc. 87 (1985), 779-785.

6. J. P. Kahane and W. Želazko, A characterization of maximal ideals in commutative Banach algebras, Studia Math. 29 (1968), 339-343.

7. Y. Katznelson, An introduction to harmonic analysis, Dover, 1968.

8. N. V. Rao, Closed subspaces of finite codimension in regular selfadjoint Banach algebras (preprint).

9. C. R. Warner and R. Whitley, A characterization of regular maximal ideals, Pacific J. Math. 30 (1969), 277-281.

10. 263-267.

11. O. Zariski and P. Samuel, Commutative algebra, vol. I, Van Nostrand, 1958.

Department of MAthematics, University of Toledo, Toledo, OHio 43606 (Current address of N. V. Rao)

Current address (R. V. Garimella): Department of Mathematics and Statistics, Northwest Missouri State University, Maryville, Missouri 64468 